\title{
$200 \mathrm{~km}$ fiber-loop Brillouin distributed fiber sensor using bipolar Golay codes and a three-tone probe
}

\author{
Zhisheng Yang*a,b, Marcelo A. Soto ${ }^{\mathrm{a}}$, Luc Thévenaz ${ }^{\mathrm{a}}$ \\ ${ }^{a}$ EPFL, Swiss Federal Institute of Technology, Institute of Electrical Engineering, \\ SCI-STI-LT Station 11, CH-1015 Lausanne, Switzerland; \\ ${ }^{\mathrm{b}}$ Permanent address: State Key Laboratory of Information Photonics \& Optical Communications, \\ Beijing University of Posts and Telecommunications, Beijing 100876, China. \\ *Email: zhisheng.yang@epfl.ch
}

\begin{abstract}
Aiming at taking full advantage of bipolar codes, a method using a three-tone probe is proposed to alleviate the probe power limitation imposed by pump depletion in Golay-coded Brillouin distributed fiber sensors. Experimental results validate the technique, which reduces significantly the measurement distortions induced by the gain/loss unbalance resulting from pump depletion/amplification. The method supports a probe power increment of more than $12.5 \mathrm{~dB}$, resulting in low-uncertainty measurements $(<0.9 \mathrm{MHz})$ at a real $100 \mathrm{~km}$ distance, using a $200 \mathrm{~km}$-long fiber loop and $2 \mathrm{~m}$ spatial resolution. The method is evaluated with a record figure-of-merit of 380’000.
\end{abstract}

Keywords: Distributed fiber sensors, stimulated Brillouin scattering, optical pulse coding, Golay codes

\section{INTRODUCTION}

Distributed optical fiber sensors based on Brillouin optical time-domain analysis (BOTDA) ${ }^{1,2}$ provide reliable solutions for distributed temperature and strain monitoring. The possibility to reach very long sensing ranges is one of the most attractive features of BOTDA sensing owing to its unique ability to perform high-quality measurements over several tens of $\mathrm{km}^{2}$. One of the main limitations on the sensor performance is imposed by the fiber losses, which highly reduce the pump and probe powers at very long sensing ranges, thus leading to a decay of the signal-to-noise ratio (SNR) of the sensor response $^{2}$. In order to provide high SNR measurements, a high pump power can be used. However, the peak pump power cannot be increased indefinitely due to the onset of nonlinear effects, such as to modulation instability ${ }^{3}$, which prematurely depletes the pump, limiting its power to about $100 \mathrm{~mW}$. To overcome this limitation, solutions such as distributed Raman amplification $^{4}$ and optical pulse coding ${ }^{5}$ have been proposed, providing a huge improvement in the sensor performance ${ }^{2}$, especially when both techniques are combined in the same system ${ }^{6,7}$, This combination has led to the best performance so far reported in a BOTDA sensor ${ }^{7}$, rated with a record figure-of-merit (FoM) ${ }^{2}$ of 300'000.

Among different types of codes applied to Brillouin distributed fiber sensing ${ }^{5-9}$, bipolar Golay codes offer the highest SNR enhancement, being $3 \mathrm{~dB}$ over traditionally-used unipolar codes ${ }^{8}$. In this paper, serious and very specific detrimental effects resulting from pump depletion in bipolar coded BOTDA sensors are identified. In order to increase the robustness of bipolar codes against pump depletion issues, a novel three-tone probe scheme is proposed, allowing for at least $12.5 \mathrm{~dB}$ probe power improvement when compared to existing implementations ${ }^{8}$. Experimental results here demonstrate the feasibility of the proposed method to reach a sensing range of $100 \mathrm{~km}$ (real remoteness) in a $200 \mathrm{~km}$-long fiber loop ${ }^{7,9}$, with $2 \mathrm{~m}$ spatial resolution and $0.9 \mathrm{MHz}$ frequency uncertainty when using 1000 averages per trace. To the best of our knowledge, results reported in this paper reach an unmatched FoM of 380’000.

\section{PRINCIPLE}

Recently, bipolar Golay codes (i.e. pulse sequences containing ‘ \pm 1 ’ elements) have been proposed for long-range BOTDA sensing $^{8}$. The reported implementation takes advantage of the Brillouin gain/loss processes in a BOTDA system: while pump pulses at frequencies higher than the probe represent the ' +1 ' code elements (inducing Brillouin gain), pump pulses at lower frequencies represent the ' -1 ' code elements (inducing Brillouin loss) ${ }^{8}$. Golay codes require launching into the fiber two bipolar pulse sequences, whose autocorrelations have complementary sidelobes that mutually cancel out when they are summed up. In an ideal condition, the autocorrelation performed during the decoding process generates a single correlation peak, with a temporal width equal to the individual pulse width, as shown by the red line in Fig. 1(a). This

24th International Conference on Optical Fibre Sensors, edited by Hypolito José Kalinowski, José Luís Fabris, Woitek J. Bock, Proc. of SPIE Vol. 9634, 96340J · C 2015 SPIE CCC code: $0277-786 X / 15 / \$ 18 \cdot$ doi: $10.1117 / 12.2195256$ 
autocorrelation function (resulting from the decoding process) can be interpreted as the temporal shape of the pump interrogating the local Brillouin gain along the fiber. Unfortunately, in real long-sensing range scenarios, coded pump pulses can experience a significant Brillouin depletion/amplification as they propagate along the fiber, thus leading to a serious power unbalance between the two pump spectral components. Consequently, the perfect Brillouin gain/loss compensation required for the proper operation of bipolar codes cannot be maintained, giving rise to additional sidelobes in the autocorrelation function, as shown by the blue and green lines in the inset of Fig. 1(a). These sidelobes, shown in the figure for $10 \%$ (blue) and $20 \%$ (green) depletion, contain the Brillouin gain/loss information from different positions (i.e. from the entire fiber section covered by the autocorrelation function), distorting the spatial information contained in the decoded traces. If the amplitude of the sidelobes is very small, most of the Brillouin interaction retrieved by the decoding can be attributed to the power of the main correlation peak, leading to correctly decoded BOTDA traces. However, when the integral of the energy confined in the sidelobes approaches the energy in the main correlation peak (e.g. $<10 \mathrm{~dB}$ difference), the measured local Brillouin gain spectrum (BGS) associated to a given fiber location will be highly affected by the BGS of neighboring fiber sections, screening the real local information contained in the measurements. Fig. 1(b) shows the power difference (in dB scale) between the main correlation peak and sidelobes as a function of the depletion level. It can be seen that with a pump depletion/amplification higher than $12.6 \%$, the contribution from the correlation sidelobes would completely dominate, resulting in a system having an equivalent spatial resolution equal to two-fold the total code length (being typically in the kilometer scale). Considering a power margin of $10 \mathrm{~dB}$ between the main autocorrelation peak and sidelobes, the system only tolerates a pump depletion lower than $1.3 \%$. This means that a very low probe power has to be used to ensure that bipolar Golay-coded BOTDA traces can be correctly decoded; however, this will impose a severe penalty to the SNR, cancelling out the benefits of bipolar coding.
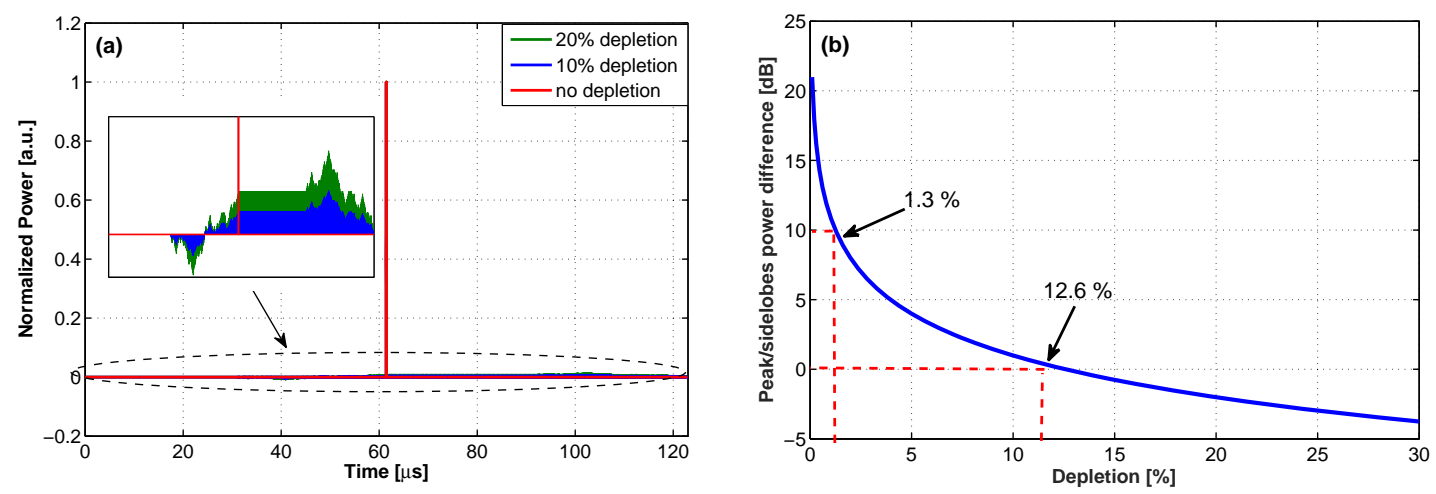

Figure 1. (a) Autocorrelation function of bipolar Golay codes in case of no depletion (red curve), $10 \%$ depletion (blue curve) and 20\% depletion (green curve). Inset: zoom of correlation sidelobes over the doubled code length. (b) Power difference (in $\mathrm{dB}$ scale) between the main correlation peak and sidelobes as a function of depletion level.

To mitigate the impact of the correlation sidelobes resulting from depletion while maintaining high probe powers, a novel implementation based on a three-tone probe scheme is proposed in this paper. In this new scheme the probe wave consists of three frequency components of equal intensity, as illustrated in Fig. 2. While the central spectral component corresponds to the coded probe signal that is detected, the upper and lower sidebands compensate for the depletion/amplification affecting the pump pulses. This way, additional probe waves are actually expected to reduce significantly the power unbalance between the two pump spectral components even at long sensing ranges. This would lead to a decoding process generating a strong main correlation peak and low-amplitude sidelobes. As a consequence, the probe power launched into the fiber can be significantly raised, offering the possibility to monitor extended fiber sensing ranges.

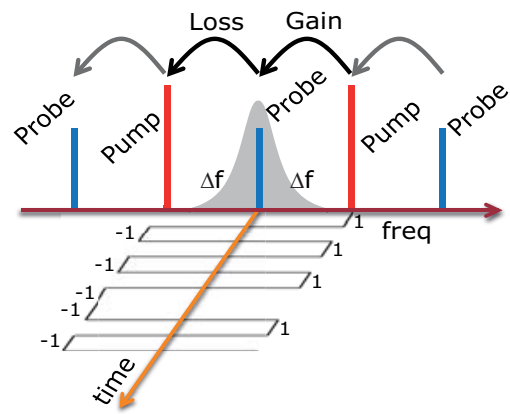

Figure 2. Principle of bipolar coded BOTDA system with 3-tone probe.

\section{EXPERIMENTAL RESULTS AND DISCUSSION}

A long-range BOTDA sensor using bipolar Golay codes and a three-tone probe has been set up as shown in Fig. 3. The light from a distributed feedback laser (DFB) is split into pump and probe branches using an optical splitter. In the pump branch, the light is injected into a high extinction ratio $(\approx 40 \mathrm{~dB})$ electro-optic modulator (EOM1), generating a carriersuppressed double-sideband signal. A $10 \mathrm{GHz}$ programmable wavelength-selective filter (WSF) is used to separate the two 
sidebands, which are intensity-modulated to generate 512-bit return-to-zero Golay-coded sequences using EOM2 and EOM3, driven by arbitrary waveform generators (AWG). The pulse width is set to $20 \mathrm{~ns}$ (corresponding to $2 \mathrm{~m}$ spatial resolution). Pump pulses at the two spectral components are properly synchronized and recombined with the same power using a coupler. Another modulator (EOM4) is then used to gate the code and to double the pump extinction ratio. After a polarization alignment, pulse sequences

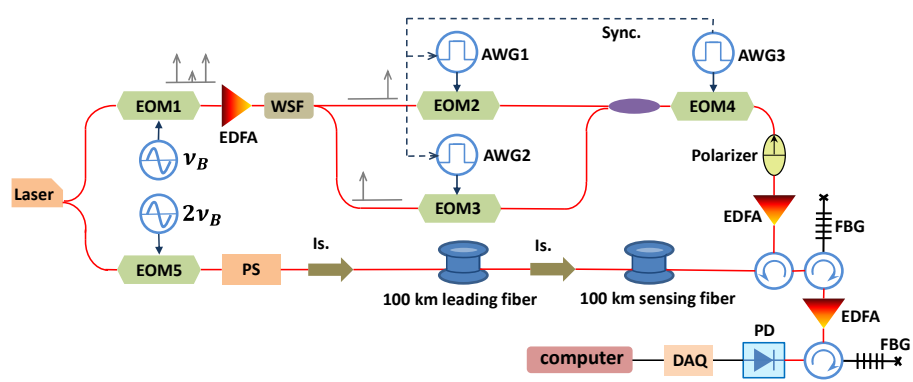

Figure 3: Experimental setup. containing the two pump spectral components are amplified up to $18 \mathrm{dBm}$ peak power and launched into a $100 \mathrm{~km}$-long sensing fiber made of four drums of fiber having similar Brillouin frequency shifts (BFS). In the other branch, a probe wave with 3 spectral tones is generated by intensity modulation using EOM5 biased at the quadrature transmission point. A polarization switch (PS) is then used to minimize the impact of the polarization-dependent Brillouin gain. The threetone probe is carried over a $100 \mathrm{~km}$-long leading fiber through an isolator. In the receiver, two tunable narrowband fiber Bragg gratings (FBG), centered at the optical carrier frequency, are used to filter out the two additional probe sidebands and the Rayleigh backscattering originating from the pump. An EDFA is used between the two FBGs as a linear-gain preamplifier. The signal is then detected by a $125 \mathrm{MHz}$ photodetector (PD) connected to a fast data acquisition (DAQ) system.

To verify the robustness of the proposed scheme, the pump depletion levels are experimentally compared in bipolar Golaycoded BOTDA sensors using a single-tone probe and a three-tone probe. The residual coded pump pulses after propagating along the $100 \mathrm{~km}$ sensing fiber are shown in Fig. 4(a) for the two cases: single-tone probe (solid blue line) and three-tone probe (dashed red line), when a power of $-11 \mathrm{dBm} /$ tone is launched into the sensing fiber. As it can be seen, there is no evident pump depletion in the three-tone probe scheme; however, the residual pump is clearly depleted by $10.7 \%$ in the conventional single-tone system. To evaluate the probe power enhancement provided by the proposed method, the depletion level for different probe powers has been measured. It was verified that the use of a three-tone probe results in negligible pump depletion even with probe powers in the $\mathrm{mW}$ level. In order to account for possible power fluctuations between the three probe spectral components in a real operating scenario, a $0.5 \mathrm{~dB}$ power difference between the optical carrier and the two sidebands was deliberately introduced. Fig. 4(b) shows that for a given level of depletion, the proposed three-tone probe technique offers an average $12.5 \mathrm{~dB}$ improvement to the input probe power permitted by the system.
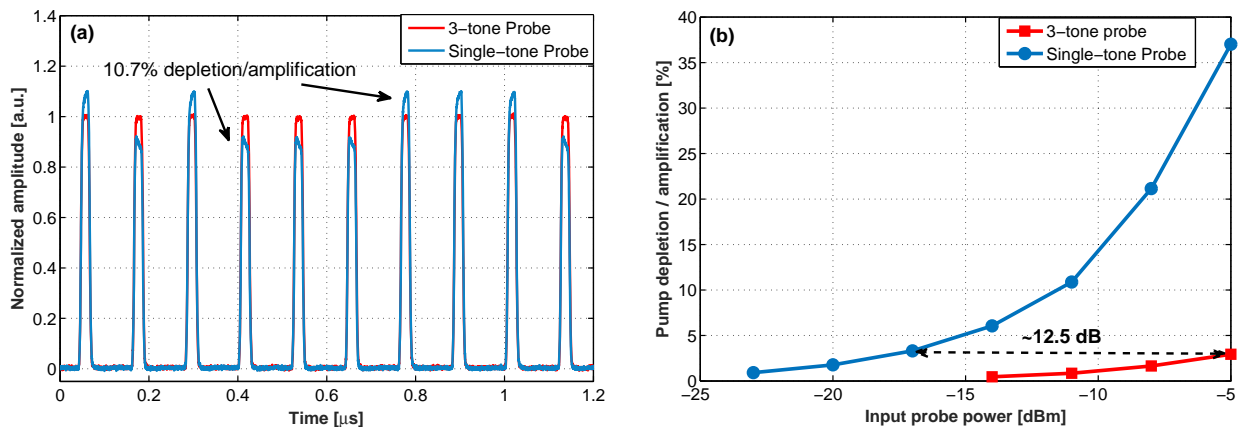

Figure 4. Comparison between Golay-coded BOTDA sensors with single-tone probe and three-tone probe (a) Normalized amplitude of bipolar Golay-coded pulses after propagation along $100 \mathrm{~km}$-long fiber. (b) Level of depletion/amplification as a function of the input probe power (considering a $0.5 \mathrm{~dB}$ power unbalance between the 3 spectral components).

Based on the results reported in Fig. 4(b), long-range BOTDA measurements with no distortions can be obtained with a three-tone probe having less than $-9.4 \mathrm{dBm} /$ tone, thus keeping the pump depletion below $1.3 \%$. In this case an optimum probe power of $10 \mathrm{dBm} /$ tone is launched into the leading fiber, resulting in $-11 \mathrm{dBm} /$ tone entering into the sensing fiber. Fig. 5(a) shows the BFS profile obtained along the $100 \mathrm{~km}$ sensing fiber with 1000 averaging per trace. Calculating the standard deviation of the BFS, the frequency uncertainty has been found to be $0.9 \mathrm{MHz}$ at $100 \mathrm{~km}$ distance. Note that the thickness of the BFS trace in the figure is only due to the coiling strain in the fibers, inducing oscillations of the BFS along the entire sensing range. Thus, taking full benefit of the high coding gain of bipolar Golay codes (13.5 dB for 512 bits) ${ }^{8}$ and of the enhanced probe power provided by the three-tone probe, the system can compensate for $\sim 42 \mathrm{~dB}$ fiber loss along the $200 \mathrm{~km}$-long loop ( $21 \mathrm{~dB}$ in the sensing fiber), without the need of distributed optical amplification. This has led to a record sensing performance, culminating to a FoM of 380’000. For comparison, the BFS obtained using a single-tone 

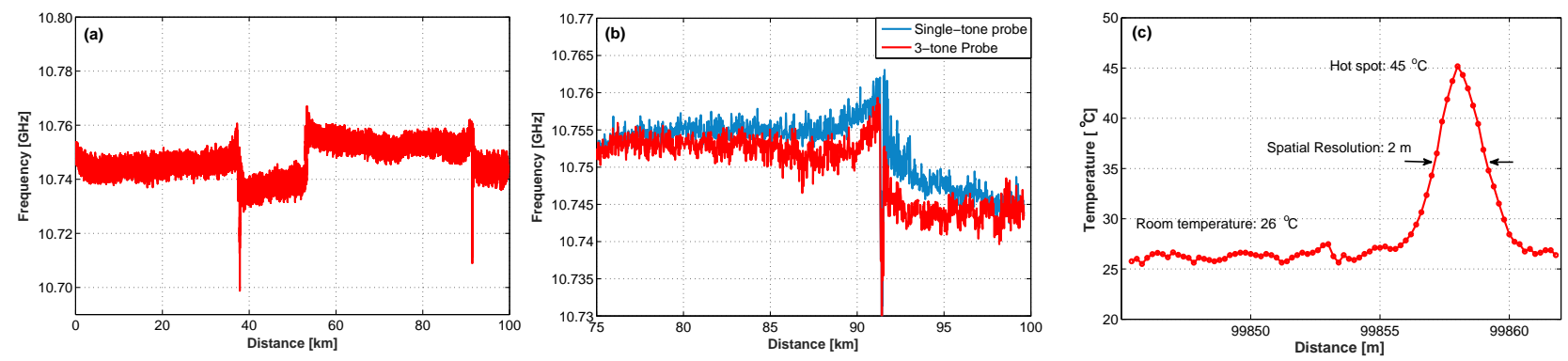

Figure 5. (a) BFS profile along $100 \mathrm{~km}$ fiber obtained by a bipolar Golay-coded BOTDA sensor using a three-tone probe. (b) BFS profiles along the last $25 \mathrm{~km}$ range, comparing the use of a single-tone probe and a three-tone probe. (c) Demonstration of a $2 \mathrm{~m}$-long hotspot detection at $100 \mathrm{~km}$ distance (real remoteness from the sensor)

probe is shown in Fig. 5(b); this shows an error of more than $5 \mathrm{MHz}$ in the BFS estimation around the transition zone between the two last fiber drums. This BFS error can actually be explained by the high pump depletion (10.7\%) and large autocorrelation sidelobes (covering a fiber section of $\sim 12 \mathrm{~km}$ ) resulting from the single-tone probe. Finally, to check the real spatial resolution of the implemented system, $2 \mathrm{~m}$ of fiber at the end of the $100 \mathrm{~km}$ sensing range have been heated up to $45^{\circ} \mathrm{C}$, while the rest of the sensing fiber has been maintained at ambient temperature $\left(26^{\circ} \mathrm{C}\right)$. Fig. 5 (c) shows the measured hotspot, revealed by a Brillouin frequency variation of $19 \mathrm{MHz}$ along $2 \mathrm{~m}$ of fiber. This result demonstrates the capabilities of the system to provide a metric spatial resolution at $100 \mathrm{~km}$ distance with no distortion and no detrimental effects resulting from pump depletion.

\section{CONCLUSION}

In this work a scheme based on a three-tone probe has been proposed and experimentally validated to reduce specific limiting and detrimental effects resulting from pump depletion in bipolar coded-BOTDA sensors. The method offers several advantages: $i$ ) possibility to use codes offering high SNR enhancement, ii) mitigation of pump depletion issues specific to bipolar codes, and iii) support of high probe powers (more than $12.5 \mathrm{~dB}$ enhancement with respect to the stateof-the-art based on a single-tone probe), which is only limited by the onset of amplified spontaneous Brillouin scattering. The combination of all these advantages has allowed compensating $42 \mathrm{~dB}$ of fiber loss in a $200 \mathrm{~km}$-long loop scheme (representing a $21 \mathrm{~dB}$ dynamic range in the BOTDA traces), with no need of distributed optical amplification. To the best of our knowledge the experimental validation of the proposed method represents a record figure-of-merit of 380’000.

The authors acknowledge the support from the Swiss Commission for Technology and Innovation (Project 13122.1) and Z. Yang is thankful to the China Scholarship Council for supporting his stay at EPFL.

\section{REFERENCES}

[1] Horiguchi, T., et al., “Development of a Distributed Sensing Technique Using Brillouin Scattering,” J. Lightwave Technol. 13(7), 1296-1302 (1995)

[2] Soto, M. A. and Thévenaz, L., "Modeling and evaluating the performance of Brillouin distributed optical fiber sensors," Opt. Express 21(25), 31347-31366 (2013)

[3] Alem, M., Soto, M. A. and Thévenaz, L., "Modelling the depletion length induced by modulation instability in distributed optical fibre sensors,” Proc. of SPIE 9157, 91575S (2014).

[4] Rodríguez-Barrios, F. at al. "Distributed Brillouin Fiber Sensor Assisted by First-Order Raman Amplification," J. Lightwave Technol. 28(15), 2162-2172 (2010)

[5] Soto, M. A., Bolognini, G., Di Pasquale, F. and Thévenaz, L., "Simplex-coded BOTDA fiber sensor with $1 \mathrm{~m}$ spatial resolution over a $50 \mathrm{~km}$ range,” Opt. Lett. 35(2), 259-261 (2010)

[6] Soto, M. A., at al., "Simplex-Coded BOTDA Sensor Over 120 km SMF with $1 \mathrm{~m}$ Spatial Resolution Assisted by Optimized Bidirectional Raman Amplification,” IEEE Photon. Technol. Lett. 24(20), 1823-1826 (2012)

[7] Soto, M. A., et al., "Extending the Real Remoteness of Long-Range Brillouin Optical Time-Domain Fiber Analyzers,” J. Lightwave Technol. 32(1), 152-162 (2014)

[8] Soto, M. A., Le Floch, S. and Thévenaz, L., "Bipolar optical pulse coding for performance enhancement in BOTDA sensors,” Opt. Express 21(14), 16390-16397 (2013)

[9] Le Floch, S., el al., "Novel Brillouin Optical Time-Domain Analyzer for Extreme Sensing Range Using High Power Flat Frequency Coded Pump Pulses,” To be published in J. Lightwave Technol. DOI:10.1109/JLT.2014.2365213 